\title{
La paridad participativa propuesta por Nancy Fraser: ¿Una radicalización de la democracia?
}

\author{
Participatory Parity Proposed by Nancy Fraser: \\ A Radicalization of Democracy?
}

MARTA VAAMONDE GAMO (Universidad de Alcalá)

Artículo recibido: 10 de enero de 2019

Solicitud de revisión: 18 de abril de 2019

Artículo aceptado: 16 de mayo de 2019

Vaamonde, Marta (2019). La paridad participativa propuesta por Nancy Fraser: ¿Una radicalización de la democracia? Recerca. Revista de Pensament i Análisi, 24(2), pp. 129150.

\section{Resumen}

Este artículo recoge la propuesta de Nancy Fraser de paridad en la participación como criterio que integra las reivindicaciones más valiosas de otras corrientes feministas con el objetivo de ampliar la justicia social en nuestras sociedades neoliberales. Asimismo, muestra los límites de su formulación: Fraser considera los condicionantes sociales que permitirían la realización de la paridad en la participación, pero no analiza en profundidad los condicionantes subjetivos de los que también depende. Por último, se sugiere superar esos límites ampliando el ideal normativo de Fraser con el pragmatismo de Dewey. El objetivo es visualizar aspectos de la justicia ocultos en la propuesta de Fraser en pro de una radicalización democrática.

Palabras clave: paridad en la participación, pragmatismo, radicalización democrática.

Abstract

This article describes Nancy Fraser's proposal of participatory parity as a criterion for the integration of the most valuable claims of other feminist schools with the goal of increasing social and gender justice within our neoliberal societies. At the same time, it shows its limits: Fraser examines the social constraints that would allow for the implementation of participatory parity, however it lacks a thorough analysis of the subjective constraints on which it also depends. Lastly, it aims to overcome those limits extending Fraser's normative ideal with Dewey's pragmatism. The main goal is to highlight aspects of justice hidden in Fraser's proposal towards a democratic radicalization. 
Key Words: participative parity, critic feminism, pragmatism, identification, democratic radicalization.

\section{INTRODUCCIÓN}

Una de las propuestas feministas más interesantes del panorama actual, a mi modo de ver, es la paridad participativa de Nancy Fraser. Con este principio normativo pretende guiar las reivindicaciones feministas hacia una radicalización de la democracia. Por radicalizar la democracia entiende ampliar el concepto de justicia para extender su alcance político en las sociedades neoliberales de nuestros días. A pesar del interés de su propuesta cabría preguntarse: ¿hasta qué punto su planteamiento amplía la noción de justica de otras corrientes feministas? y ¿qué límites de su propuesta teórica frenan el alcance emancipador de su iniciativa feminista y limitan la radicalización democrática?

Fraser considera acertadamente que la crisis general del sistema capitalista en su forma actual - globalizadora y neoliberal - presenta nuevos retos al feminismo. El neocapitalismo ha ocasionado sus propias crisis (ecológica, demográfica y política), porque la producción de capital se asienta en unas condiciones de reproducción sujetas a explotación y expropiación. Se explotan recursos naturales que se consideran erróneamente como ilimitados y las tareas de cuidado recaen mayoritariamente en mujeres inmigrantes en régimen de explotación. El endeudamiento, además, es característico del capitalismo financiero y los Estados se preocupan más en saldar su deuda que en el bienestar de sus ciudadanos. Esas crisis están estrechamente relacionadas entre sí y, por eso, la lucha por la igualdad de género debe relacionarse con la lucha ecológica y la lucha racial. En resumidas cuentas, el feminismo no debe alentar a un número reducido de mujeres a alcanzar altas cotas de poder en unas estructuras capitalistas injustas. Fraser propone un feminismo del $99 \%$, es decir, que atienda a los intereses de la mayoría de las mujeres y que, junto con otros movimientos emancipadores, sea capaz de transformar profundamente el neocapitalismo.

El marco teórico más apropiado para que el feminismo pueda cumplir con este objetivo es, de acuerdo con Fraser (2011: 158-159), el pragmatismo. El pragmatismo tiene indudables ventajas respecto a otros paradigmas feministas. En primer lugar, sitúa la teoría en los contextos sociales concretos y múltiples. Esto significa que hay que entender la jerarquía y las luchas de género en todos 
los ámbitos de la vida social en los que aparece: familia, sociedad civil, estructuras económicas y aparatos del Estado, etc. Además, permite entender que la jerarquía de género está atravesada por otras estratificaciones de poder (raciales, étnicas, religiosas etc.), es decir, no aísla las luchas de género de otros movimientos emancipadores como ocurre con otros planteamientos feministas de corte lacaniano y posestructuralista. Asimismo, el carácter situado de la teoría feminista le permite atender mejor la pluralidad cultural, social y sexual de las mujeres.

Pero, además, el enfoque pragmatista de Fraser vincula directamente su teoría feminista con la práctica social. El objetivo de la teoría, lo que calibra su valor, es su capacidad para proyectar ideales utópicos, falibles y revisables, que puedan guiar la transformación social (Fraser, 2011: 159).

Este ideal crítico y social de su feminismo pragmatista lo distingue del pragmatismo de Richard Rorty y lo acerca al deweyano. En 1990 Rorty fue ponente de las Tanner Lecture on Human Values con una sesión titulada «Feminismo y pragmatismo». La réplica estuvo a cargo de Fraser con la lección: «De la ironía a la profecía y de la profecía a la política». Tanto para Fraser como para Rorty los discursos no tienen ningún fundamento trascendental, sino que se generan históricamente. Esta idea permite liberar a las mujeres de determinaciones reificadas de su identidad para que sean ellas mismas las que se autodesignen. Ambos entienden la innovación lingüística como un elemento indispensable en la emancipación, pues como afirma Marta Palacio siguiendo a Fraser:

Cada campo de la vida social está impregnado de prácticas simbólicas, y cada acción se realiza desde el interior de un horizonte de significados e interpretaciones culturales ( $\mathrm{Pa}$ lacio, 2018: 16).

Sin embargo, esta construcción de la identidad adopta en Rorty un carácter liberal y estético, mientras que en Fraser tiene una dimensión social y política. Para Rorty la emancipación consiste en la creación de sí a través de las innovaciones del lenguaje en unas estructuras sociales liberales. Las feministas son como profetas que nos han mostrado el camino de la emancipación. Para Fraser las innovaciones en los actos de habla a través de los cuales se construye la identidad de las mujeres son sociales y políticas, las han llevado a cabo gru-

1 Puede encontrarse su debate en Janack (2000). 
pos feministas con un claro objetivo político y social. La identidad actual de la mujer, como señala Palacio:

Constituye ese trabajo colectivo y de democratización que el feminismo ha llevado a cabo hasta lograr identificar en la esfera pública una forma de identidad feminista (Fraser, 2004) en la que el término mujer supone la pluralidad y la heterogeneidad social (Palacio, 2018: 18).

Esta dimensión social y política del pragmatismo vincula a Fraser con John Dewey. Ambos entienden el feminismo como una concreción del ideal democrático, relacionado, por tanto, con otros ejes sociales de dominación y cuyo objetivo es la transformación de las instituciones sociales desigualitarias. Asimismo, afirman que la democracia es un ideal que consiste en la participación en los diálogos sociales que deben ordenar todas las interacciones. Por tanto, Dewey coincidiría con Fraser en que la paridad en la participación es un criterio adecuado para determinar la justicia social. Sin embargo, el pragmatismo de Dewey permite ampliar la interpretación de este principio, lo que resulta útil para avanzar hacia la radicalización democrática y feminista.

Desde el pragmatismo de Dewey, la paridad en la participación no solo es un principio normativo que permite guiar las luchas sociales y los movimientos emancipatorios, sino que adquiere un sentido moral. Se trata de un principio vital que permite orientar la tarea contingente y concreta de ampliar nuestra autonomía. El análisis de Dewey permite así vislumbrar no solo las medidas institucionales requeridas para lograr la paridad en la participación, sino también las personales.

Examinaré, en primer lugar, la propuesta de la paridad participativa de Fraser para comprobar que cumple lo que promete: la radicalización de la democracia a través de una noción ampliada de justicia. En segundo lugar, y tras sopesar sus ventajas y límites en relación a las críticas de Judith Butler y Axel Honneth, presentaré, en diálogo con Fraser, una forma alternativa de radicalización democrática desde el pragmatismo deweyano.

\section{PARIDAD EN LA PARTICIPACIÓN}

Fraser entiende la justicia como paridad en la participación, que supone que todos los miembros mayores de edad de una institución puedan participar como interlocutores plenos en su funcionamiento. Adapta la tríada weberiana 
de clase, estatus y partido a tres tipos de obstáculos a la paridad participativa en las sociedades capitalistas: los derivados de la injusta distribución, el mal reconocimiento y la falta de representación (Fraser, 2015: 31). Puesto que están relacionados entre sí, pero no hay ninguno fundamental al que puedan reducirse los otros, representan tres dimensiones de la justicia que requieren diversas respuestas políticas.

La política de la redistribución y la política del reconocimiento se distinguen, según Fraser (2011: 294), en tres aspectos clave. En primer lugar, en su concepción de la injusticia. La política de la redistribución se centra en las injusticias que define como socioeconómicas y presume que están arraigadas en lo socioeconómico como la explotación, la privación y la marginación económica. La política del reconocimiento apunta a las injusticias que considera culturales y supone arraigadas en los patrones sociales de representación, interpretación y comunicación, como la dominación cultural, la falta de respeto y el no reconocimiento.

En segundo lugar y como consecuencia, los dos enfoques presentan distintos remedios contra la injusticia. Para la política de la redistribución, señala Fraser:

El remedio es la reestructuración socioeconómica, por ejemplo, redistribuir la renta, reorganizar la división del trabajo [...] para la política del reconocimiento, por el contrario, el remedio para la injusticia es el cambio cultural o simbólico (Fraser, 2011: 294).

Por ejemplo, medidas políticas destinadas a revalorizar las identidades, la diversidad cultural o transformar patrones sociales de representación, interpretación y comunicación.

En tercer lugar, las dos orientaciones políticas asumen diferentes concepciones de los colectivos que padecen injusticias. Para la política de redistribución, son clases que se definen por su relación con el mercado o los medios de producción - minorías étnicas explotadas, inmigrantes, mujeres que tienen un sueldo inferior y doble jornada de trabajo-. Para la política del reconocimiento, las víctimas de la injusticia no se definen por las relaciones de producción, sino por su menor estima, honor y prestigio en relación a otros grupos sociales.

Un ejemplo de la diferencia entre las injusticias por la distribución y las injusticias por el mal reconocimiento se encuentra en la discriminación de afroamericanos en EE. UU. y la discriminación de los judíos en la Alemania nazi. En el primer caso, no se busca la aniquilación de los negros, sino mantenerlos como una clase subordinada sujeta a explotación por parte de empresas 
o terratenientes. En el segundo caso, se perseguía la exterminación de los judíos como identidad cultural en respuesta a grupos ideológicos (Fraser, 2011: 294).

En el escenario político global, Fraser añade otra dimensión: la representación. Si la redistribución hace referencia a la economía y el reconocimiento hace referencia a la cultura, la representación hace referencia a la política en un sentido más específico. Tanto la redistribución como el reconocimiento son categorías políticas, pues implican luchas de poder, pero la representación tiene una referencia política más concreta: la jurisdicción constitutiva de una comunidad política y las normas de decisión. La representación se centra en cuestiones como la pertenencia, qué individuos están incluidos o excluidos de qué comunidades, y también en cuestiones de procedimiento, cómo los individuos presentan y dirimen sus reivindicaciones. Tanto la pertenencia como las normas de procedimiento deben ser justas y pueden acarrear injusticias.

Esta dimensión política de la justicia no puede reducirse a la distribución o al reconocimiento porque da lugar a injusticias específicas derivadas de la organización política de la sociedad distinta de la estructura de clase y del orden de estatus. Aunque diferentes, esas dimensiones de la injusticia se relacionan. La dimensión política establece el espacio en el que los individuos pueden dirimir sus reivindicaciones distributivas y de reconocimiento.

La injusticia política característica es la falta de representación. No solo derivada del procedimiento, es decir, de que las normas de decisión afectan a la paridad en la participación, sino también del marco en el que se dirimen las cuestiones políticas. Estos marcos a veces excluyen a ciertos individuos afectados que no pueden hacer valer sus reivindicaciones ni de distribución ni de reconocimiento, o puede haber individuos excluidos de cualquier marco, por ejemplo, los solicitantes de asilo. El problema del marco de justicia es uno de los más acuciantes en un mundo globalizado.

Las categorías de Fraser (redistribución, reconocimiento y representación) no solo son analíticas, es decir, no solo permiten visualizar distintas dimensiones de la justicia, sino que, desde su punto de vista, se corresponden a distintos momentos del cambio social y del feminismo de segunda ola.

En el momento culminante del Estado del bienestar, el objetivo de las reivindicaciones era fundamentalmente la redistribución (Fraser, 2015: 230). Las feministas de los años setenta influidas por el marxismo centraban sus esfuerzos en la economía política y el trabajo. Criticaban la desatención marxista de la reproducción e interpretaban el género como una clase (Benhabib y Cornell, 1990: 14). 
Junto al feminismo de corte marxista centrado en la explotación de la mujer en la reproducción, se empezaron a desarrollar nuevas corrientes feministas en diálogo con el psicoanálisis (Amorós y De Miguel, 2010: 29). En el mundo anglófono comenzaron a conceptualizar el género como una identidad. En Europa los lacanianos rechazaban el término relaciones de género por excesivamente sociológico y lo reemplazaban por el de diferencia sexual relacionada con la construcción de la subjetividad y el orden simbólico. Tanto el feminismo directamente inspirado en el marxismo como el procedente del psicoanálisis tenían como objetivo ampliar críticamente la perspectiva marxista (Fraser, 2015: 189). Junto a movimientos que luchaban por la igualdad de los negros en EE. UU., las luchas antiimperialistas y anticolonialistas, por ejemplo, contra la guerra del Vietnam, contribuyeron a ampliar la política democrática de los Estados del bienestar.

En un segundo momento, en un contexto social distinto, el feminismo cambió. La globalización, la caída del comunismo, la crisis del Estado del bienestar, el triunfo de la derecha en el Reino Unido y EE. UU. motivaron el auge del neoliberalismo de los ochenta. Los ideales socialdemócratas perdieron fuerza y el feminismo dio un giro cultural. La mayoría de las feministas concibieron el género como una identidad o construcción cultural. Este cambio en el concepto de género supuso un cambio también en su forma de entender la justicia y en sus reclamaciones políticas. Su objetivo era lograr un reconocimiento respetuoso de la diferencia de género. Se vincularon a movimientos que buscaban también un reconocimiento de la diferencia étnica, sexual o racial; reclamaban que la integración social no supusiera asimilación a las normas mayoritarias. En el entorno académico, la teoría de género se convirtió en una rama de los estudios culturales. En resumidas cuentas, se debilitaron los lazos históricos del feminismo con la teoría social y la economía política. Se produjo lo que Fraser denomina un desplazamiento de la distribución al reconocimiento (Fraser, 2000: 326 ).

Los resultados de la política feminista del reconocimiento fueron ambivalentes (Fraser, 2015: 21).

Por una parte, ampliaron el sentido de la justicia otrora centrado en el trabajo y la distribución, que se extendió a la falta de reconocimiento de ciertos grupos en la representación, la interpretación y la interacción social. En los años ochenta y noventa, el feminismo además logró una amplia difusión social a nivel global.

Por otra, las luchas por el reconocimiento se desviaron de la crítica político-económica del capitalismo. La consecuencia fue que el feminismo asentó 
vínculos con el neoliberalismo. Se produjo lo que Fraser considera cuatro paradojas históricas (Fraser, 2015: 33). La crítica feminista al economicismo socialdemócrata que fue emancipadora en el marco del Estado del bienestar ha contribuido al interés neoliberal de desviar las luchas socioeconómicas hacia la cultura. En segundo lugar, la crítica feminista al salario familiar ha servido para justificar la familia con dos proveedores en peores condiciones laborales. En tercer lugar, la crítica feminista al paternalismo del Estado del bienestar ha confluido con las críticas neoliberales a las protecciones sociales estatales. Por ejemplo, las políticas de microcréditos impulsadas por el feminismo pueden tener el efecto perverso de obviar la implicación de los gobiernos en la igualdad. En cuarto lugar, los esfuerzos por ampliar el alcance de la justicia de género más allá de los Estados nacionales, con la participación de comités feministas en las Naciones Unidas, la Unión Europea y otros órganos de dirección transnacional pueden confluir con el deseo neoliberal de evitar el control político estatal de los mercados.

Sin embargo, el capitalismo actual, neoliberal y global, ha entrado en crisis. Fraser menciona la crisis financiera, la ecológica y la reproducción social; desde mi punto de vista, habría que añadir la crisis migratoria. Como consecuencia de esta situación, el feminismo se ha internacionalizado, hay un renovado interés por la política económica y el culturalismo está ampliamente desacreditado (Fraser, 2015: 30). En tal orden de cosas, Fraser considera que las luchas feministas tienen que desarrollarse simultáneamente en tres frentes: la redistribución, el reconocimiento y la representación, que son las condiciones de la paridad participativa. Esta visión ampliada de justicia integra las dimensiones que históricamente se habían fragmentado con el fin de renovar la dimensión transformadora y política del feminismo (Fraser, 2015: 22).

La cuestión es si sus categorías de distribución, reconocimiento y representación expresan adecuadamente las luchas feministas y sus cambios históricos y si permiten integrar sus reivindicaciones y radicalizar el potencial crítico y democrático del feminismo.

\section{CRÍTICAS A LA PROPUESTA DE FRASER}

La propuesta de Fraser es realmente ambiciosa y tiene indudables ventajas. En primer lugar, intenta integrar los aspectos más valiosos de distintas reivindicaciones feministas. Atiende a distintas dimensiones de la justicia (económica, cultural y política) sin reducir unas a otras, es decir, evitando tanto el 
economicismo del feminismo marxista como el culturalismo de algunas de las corrientes del feminismo del reconocimiento.

Tiene en cuenta también el carácter intersectorial de las reivindicaciones feministas. Las luchas por la igualdad de género adquieren importantes matizaciones de raza y etnia cuando se trata de reivindicaciones de mujeres de otras etnias y no de mujeres occidentales (Femenías, 2007: 155-156).

En última instancia, Fraser pretende revitalizar el potencial crítico y transformador del feminismo, por eso presenta un interesante análisis social que responde a los problemas políticos actuales más controvertidos. Sin embargo, creo que tiene límites que frenan su pretensión de integrar las reivindicaciones feministas ampliando radicalmente la justicia.

El primero de esos límites hace referencia a la narración de la historia de la segunda ola del feminismo. Desde su punto de vista, ha habido un desplazamiento de la política de la distribución del feminismo interesado en la igualdad social al feminismo del reconocimiento de la identidad cultural. La consecuencia ha sido, en palabras de Fraser, un alejamiento feminista de la política económica. Las políticas del reconocimiento, señala, o bien se desentendían de la justa distribución, o bien la consideraban como un epifenómeno de las jerarquías de estatus. Como consecuencia, creían que las políticas para remediar el mal reconocimiento repararían indirectamente la mala distribución (Fraser, 2011: 265$)$.

Sin embargo, a mi modo de entender, no todas las reivindicaciones de la diferencia se desinteresaban de las desigualdades económicas. Algunas interpretan el orden social de género como un elemento clave del orden económico, por ejemplo, Judith Butler (Fraser, 2011: 265).

Butler cuestiona la separación de Fraser de las reivindicaciones destinadas a la redistribución de recursos, que ubica en el centro de la política económica y social, y las luchas orientadas al reconocimiento, que sitúa en el orden cultural. De acuerdo con Butler, precisamente una de las cuestiones que puso de manifiesto el feminismo socialista de los años setenta y ochenta es la importancia económica del ordenamiento político y social de la sexualidad. El emblema de la segunda ola del feminismo, «lo personal es político», expresaba esta idea. Butler afirma: «La regulación de la sexualidad se vinculaba sistemáticamente con el modo de producción apto para el funcionamiento de la economía política» (Butler, 2000: 120). Butler acaba concluyendo que la heterosexualidad es un elemento clave en la organización social capitalista y que, por tanto, las reivindicaciones de reconocimiento de la diferencia sexual tendrán el efecto de transformar la sociedad capitalista. 
Fraser señala la diferenciación de esferas en el capitalismo contemporáneo (Fraser, 2015: 209). La racionalidad de los sistemas económicos, del mercado, no coincide con los patrones de valor que rigen las interacciones comunicativas en el mundo de la vida y, así, un varón blanco en paro puede tener problemas de renta aunque no tenga problemas de reconocimiento y, al revés, un homosexual puede tener problemas de reconocimiento, teniendo un buen puesto en el mercado laboral. Si bien la falta de reconocimiento y la mala distribución se relacionan, guardan una relativa independencia. Esto significa que el heterosexismo no es clave en el ordenamiento económico del capitalismo actual y que, por tanto, las luchas por el reconocimiento de la diferencia sexual no transformarán esencialmente el capitalismo (Fraser, 1997: 285). Creo que el análisis social de Fraser es más ajustado que el de Butler. El reconocimiento de la diferencia sexual no parece que pueda desmantelar al capitalismo actual.

Sin embargo, Fraser deja sin analizar un aspecto clave del análisis de Butler. Butler subraya la importancia que la organización política y social de la sexualidad tiene en la propia producción de la subjetividad, en la producción de unos sujetos que perpetúan así el orden económico e institucional que los constituye (Butler, 2007: 47-48). De hecho, desde su punto de vista, uno de los principales hallazgos del feminismo de los setenta y ochenta fue darse cuenta de que la (re)producción social dependía de la (re)producción individual. Este feminismo amplió el planteamiento marxista de la explotación de la mujer buscando en el psicoanálisis los mecanismos de sujeción de la personalidad y la forma en que esta regulación se manifestaba en los deseos sexuales (Fraser, 2011: 265).

El análisis de Fraser, tomando como punto de partida las reivindicaciones sociales y las injusticias institucionales, deja sin analizar los mecanismos mediante los cuales se configura a los sujetos que integran las instituciones. A mi modo de ver, siguiendo a Butler y Foucault (Foucault, 2015: 318; Foucault, 1990: 11), hay mecanismos de sujeción individual que un análisis social debería visibilizar.

En última instancia, y si analizamos algunas de las reivindicaciones políticas del reconocimiento, no desplazaron su enfoque de intereses económicos a intereses culturales, sino que interpretaron el papel económico de la diferencia sexual desde un planteamiento teórico distinto. Alex Honneth también se muestra crítico con la idea de Fraser del desplazamiento. A este respecto señala: 
Me parece muy inverosímil interpretar la historia del conflicto político en las sociedades capitalistas según un esquema que afirma una transición desde unos movimientos sociales basados en intereses a otros orientados a la identidad (Fraser y Honneth, 2006: 107).

Según Honneth, Fraser generaliza indebidamente como propio del desarrollo histórico de la segunda ola del feminismo un desplazamiento que habría ocurrido en EE. UU., donde la política de reconocimiento cobró fuerza, pero que no ocurrió en otros contextos, por ejemplo, Alemania, Francia o Gran Bretaña, donde las políticas laborales, ecológicas y de bienestar siguieron siendo centrales (Fraser y Honneth, 2006: 95). Por otra parte, Honneth lamenta que no tenga en cuenta los precursores históricos de los movimientos sociales de reconocimiento para que parezcan movimientos nuevos que han desplazado a las anteriores luchas sociales (Fraser y Honneth, 2006: 99).

El análisis histórico de Fraser y, por consiguiente, el problema del desplazamiento se deducen, según Honneth, de las categorías empleadas en su análisis y en las que ubica los distintos movimientos sociales. Nos encontramos así con un segundo problema, el problema de la adecuación de las distintas corrientes en su esquema categorial. Si el primer problema, el desplazamiento, ponía el énfasis en las limitaciones del análisis histórico de Fraser, el problema de la adecuación se refiere fundamentalmente a los límites de su análisis sociológico.

Fraser encuadra dentro de la política de redistribución las corrientes feministas que buscan una transformación socioeconómica como remedio de la injusticia de género. Esta orientación engloba también el liberalismo del New Deal, la socialdemocracia y el socialismo, asimismo, algunas formas de antirracismo dirigidas a la reforma socioeconómica como reparación de la injusticia racial-étnica. Entiende por política de reconocimiento no solo los movimientos sociales dirigidos a revalorizar las identidades devaluadas, por ejemplo, el feminismo cultural, el nacionalismo cultural negro y la política de identidad gay, sino también las políticas deconstructivas, como la política queer (Posada, 2014: 147), la política crítica de raza y el feminismo deconstructivo (Fraser, 2011: 293294).

Sin embargo, Honneth malinterpreta el desplazamiento como sucesión. Si bien el interés feminista se desplaza de la redistribución al desplazamiento, eso no significa que no existieran antecedentes históricos del reconocimiento. Respecto a la adecuación de incluir en una misma categoría propuestas políticas muy dispares centradas bien en la redistribución, como el liberalismo del New Deal o la socialdemocracia, bien en el reconocimiento, como la política de 
la identidad o la política queer, no significa que coincidan en lo que consideran una justa redistribución o un justo reconocimiento.

Honneth, no obstante, manifiesta un límite del análisis de Fraser al que merece la pena prestar atención. No por la alternativa que presenta, poco interesante quizá para el feminismo, pero sí por el problema al que apunta.

Fraser utiliza la paridad participativa como principio normativo para dirimir las reivindicaciones de justicia en la sociedad contemporánea. Ahora bien, las luchas sociales de las que parte no tendrían fuerza emancipadora si no gozasen de cierta adhesión por parte de los individuos. Si bien Honneth puede señalar un asunto interesante, su propuesta es problemática. Entiende que todas las experiencias de injusticia tienen su origen en el sufrimiento por el mal reconocimiento de la propia identidad. Determina entonces la identidad reconocida como el ideal ético que determina y limita su análisis social, pues las esferas sociales se corresponden con la necesidad de su reconocimiento, así como los principios normativos que deben regir cada una de esas esferas.

Además de reductivo, su planteamiento resulta abstracto. Su punto de partida son las experiencias prepolíticas de injusticia (Fraser y Honneth, 2006: 94). Sin embargo, toda experiencia de injusticia está mediatizada políticamente. Finalmente, o bien su determinación de la identidad realizada es indeterminada para poder albergar distintos modelos de vida y, en este caso, es poco operativa como principio normativo o, si es muy determinada, limita la autonomía personal y la pluralidad social. Por otra parte, las reivindicaciones feministas son emancipatorias, es decir, precisamente pretenden evitar que una determinación previa de su identidad limite la autonomía de las mujeres.

Sin embargo, podemos observar que, desde presupuestos distintos, coincide con el posestructuralismo al señalar que el análisis de Fraser no es suficientemente radical, pues parte de movimientos sociales y supone la capacidad de los sujetos para participar en ellos sin tener en cuenta los presupuestos institucionalizados que nos convierten en los sujetos que somos. Al no considerar estos procesos de construcción de la subjetividad, la teoría contribuye a su perpetuación.

Pero, ¿el dilema entre analizar las luchas sociales sin atender a los condicionantes subjetivos que perpetúan y sirven de base a los discursos sociales, por una parte, o analizar los mecanismos psicológicos de la acción sin considerar que depende de los discursos sociales en los que se ubica, por otra, es insalvable? ¿Podemos asumir un análisis de las relaciones entre los grupos sociales y la subjetividad que no caiga en la reificación de la identidad y al tiempo procure una dirección a las luchas feministas emancipatorias? 


\section{AMPLIACIÓN DE LA PROPUESTA DE FRASER DESDE EL PRAGMATISMO DE JOHN DEWEY}

Fraser, en un trabajo conjunto con Linda Nicholson, «Social Criticism Without Philosophy: An Encounter between Feminism and Postmodernism» (Fraser y Nicholson, 1988), intenta integrar teoría crítica y posestructuralismo desde un modo de teorización feminista pragmático y falibilista (Benhabib, Butler, Cornell y Fraser, 2011: 62). Podríamos hacer un ensayo semejante para conservar, desde una perspectiva pragmática que amplíe la propuesta de Fraser, el interés emancipador del feminismo crítico adoptando las críticas acerca de sus supuestos no analizados.

Una de las críticas al paradigma formulado por Fraser es que parte de los movimientos sociales que considera políticamente relevantes en la sociedad actual, sin atender a las características de los sujetos que les llevan a formar parte de esos movimientos o que les capacitarían para reclamar justicia. Su perspectiva, desde el punto de vista del feminismo posestructuralista, no analiza en profundidad los mecanismos de normalización de la subjetividad.

El enfoque de la teoría democrática y social de John Dewey de la que el feminismo se puede servir para ampliar el significado de la paridad participativa no parte ni de los movimientos sociales ni se queda en un análisis psicológico que desatiende la crítica y la transformación social capitalista, que es el temor de Fraser hacia algunas corrientes que sitúa en las políticas del reconocimiento. Dewey parte de las transacciones entre el individuo y la comunidad para entender el tipo de relaciones específicamente democráticas.

Dewey introduce el término transacción en su última obra publicada, Knowing and the Know (Hamington y Bardwell-Jones, 2012) para sustituir la palabra relación. El objetivo era recalcar su sentido radical. Una transacción es una relación en la que los términos que interactúan resultan modificados por su interacción. En el caso de las transacciones humanas, el sujeto, de acuerdo con Dewey, desarrolla su identidad y su acción por medio de sus relaciones sociales (Dewey, 1916: 4-5). La comunidad proporciona al individuo los medios para ordenar su experiencia, comprenderse a sí mismo y establecer los cauces de su acción. Sin embargo, la comunidad no determina ni anula la individualidad. Cada individuo, piensa Dewey, tiene una manera única de interactuar con su entorno (Dewey, 1934: 286).

Este comportamiento selectivo no está determinado culturalmente, sino que es un rasgo que compartimos con los seres naturales. Dewey explicaba así este sentido dinámico y relacional de la individualidad: 
La individualidad es, en un primer momento, espontánea y carente de forma; es potencialidad, una capacidad de desarrollo. Aun así, es una manera única de actuar en y con un mundo de objetos y personas. No es algo completo en sí, como un armario o un cajón secreto en un escritorio, lleno de tesoros esperando a ser concedidos al mundo. Dado que la individualidad es una manera distintiva de sentir los impactos del mundo y de mostrar sesgos preferenciales en respuesta a estos impactos, adquiere perfil y forma solo mediante transacción con condiciones reales (Dewey, 1930: 138, en Bernstein, 2010: 173).

Si bien es cierto que los factores que mueven las elecciones, los intereses, las necesidades y la visión que tenemos de nosotros mismos dependen de las transacciones del individuo con el entorno, en esas transacciones hay una selección personal que distingue a los individuos. Precisamente por eso, no solo la comunidad condiciona al individuo, sino que el individuo puede transformar la sociedad (Seigfried, 2001: 174).

Desde el feminismo deconstructivo se critica el planteamiento de Fraser porque no analiza en profundidad los condicionantes de la subjetividad. La referencia de la teoría democrática de Dewey a las transacciones permite completar el análisis de Fraser de los movimientos sociales con el análisis de los condicionantes subjetivos de las interacciones sociales.

En uno de sus trabajos más reconocidos, Human Nature and Conduct: An Introduction to Social Psychology de 1922, Dewey presenta una interpretación de todos los factores psicológicos del sujeto como funciones de la conducta, es decir, de la transacción del individuo con el medio. La referencia a las transacciones permite relacionar entre sí las distintas dimensiones de la subjetividad y, a su vez, referirlas también a las relaciones del individuo con el entorno.

Uno de los problemas del tratamiento de la subjetividad por parte del feminismo posestructuralista, el feminismo crítico o las corrientes comunitaristas es que consideran fundamental y característico de la identidad uno de sus elementos. De esta manera, pierden su relación con la conducta, es decir, su relación mutua y su vinculación con la dimensión social de la personalidad. El feminismo crítico concede una importancia fundamental a la capacidad crítica de los sujetos, por ejemplo, de las mujeres, que hace posible sus reclamaciones emancipatorias. El feminismo deconstructivo y posestructuralista recalca el papel determinante de los deseos inconscientes en el comportamiento, las corrientes comunitaristas y los lazos afectivos con la comunidad a la que históricamente pertenecemos. Dewey afirma: 
Todas estas concepciones son consecuencia natural de una falta al no reconocer que todo conocimiento, juicio y creencia representan el resultado obtenido por el funcionamiento de los impulsos naturales en relación con el medio ambiente (Dewey, 1922: 130).

La interpretación pragmática de la subjetividad no concibe ninguna de estas capacidades como sustantiva, ni como definitorias del sujeto, sino como funciones de la conducta. Por consiguiente, no hay un sujeto sustantivo detrás del comportamiento. Señala Dewey:

No hay un ser ya formado detrás de las actividades; hay actitudes, hábitos e impulsos complejos, inestables y contradictorios, que gradualmente se adaptan unos a otros, adquiriendo cierta coherencia en su configuración (Dewey, 1922: 96).²

Como resultado, es el individuo el que configura su personalidad con sus actos. La inteligencia, los deseos y la imaginación son factores que se expresan en la conducta que, al mismo tiempo, produce hábitos y actitudes que reorganizan en una u otra dirección esas capacidades y sus mutuas relaciones. Por eso, si bien es necesario el análisis social de los grupos reivindicativos, la extensión social de la paridad participativa exige disposiciones y actitudes personales.

El análisis pragmático refiere la subjetividad a las transacciones, de modo que las relaciones democráticas que mejoran y potencian las relaciones sociales también potencian el desarrollo personal y viceversa. De esta manera, si el paradigma de Fraser, la paridad participativa, sirve para visualizar y luchar contra relaciones desigualitarias, el planteamiento de Dewey permite ampliarlo atendiendo a las actitudes morales que la extensión social de este principio normativo exige.

La interpretación deweyana del desarrollo moral depende de su interpretación funcional de las capacidades humanas. Dewey entiende que la reflexión y la inteligencia tienen como función dirigir la conducta y así afirma:

La actitud racional es la disposición que resulta, no un antecedente ya formado que puede ser invocado y puesto a funcionar a voluntad [...] la «razón» no es una fuerza antecedente que pueda usarse como panacea, es la obra laboriosa del hábito y requiere que se la ejercite continuamente (Dewey, 1922: 136-137).

2 Esa coherencia no es total ni idéntica en todos los individuos, es un logro de su acción. En ocasiones, la coherencia se limita, afirma Dewey, a: «Una distribución de incompatibilidades que las mantenga en compartimentos impenetrables, dándoles turnos sucesivos de acción» (Dewey, 1922: 96). 
Lo que caracteriza a la razón es, por tanto, su cometido práctico. Por eso, lejos de oponerse, la reflexión y el impulso se complementan. La reflexión tiene un papel directivo en la conducta; tras una deliberación, determina un curso de acción. Ahora bien, la reflexión comienza cuando se rompe el automatismo de la conducta pasada, y esa ruptura viene marcada por el deseo, que, de este modo, es el motor, el impulso de la reflexión (Dewey, 1922: 16).

Si bien es cierto que visibilizar los deseos inconscientes que condicionan la acción es importante, tal y como viene desarrollando hace tiempo el feminismo psicoanalítico, el deseo no se identifica solamente con los impulsos inconscientes. Los deseos están implicados en todas las acciones, también las racionales; sin los deseos, la reflexión sería imposible. La reflexión y la afectividad se limitan cuando se separan, pero se potencian y potencian la conducta cuando se integran, que es, precisamente, el objetivo de la moralidad: no anular, sino dirigir los afectos. El resultado de esa integración es lo que Dewey denomina la buena voluntad. La buena voluntad supone también la confluencia de otras dimensiones de la conducta, en concreto, la social y la imaginativa. Dewey denomina simpatía a la racionalización de la afectividad que es posible por el concurso de la imaginación. La define como: «la porción de la estructura psicológica de una persona que le permite identificar los fines de los otros con los suyos propios» (Saito, 1996: 391).

La imaginación posibilita el crecimiento de la simpatía. En primer lugar, porque amplía su campo de aplicación, pues no solo nos permite identificarnos con los más próximos, sino con toda la humanidad; en segundo lugar, permite reconstruir imaginativamente la acción con el fin de compartir experiencias. Como apuntaban las palabras de Dewey, esta imaginación simpática es indispensable para fomentar la liberación del otro y ampliar su campo de acción, creando intereses comunes (Dewey, 1908: 128). Esa acción integrada de la emoción y la imaginación es lo que procura a la reflexión su objeto, los bienes a evaluar. Sin el concurso de la imaginación y de la simpatía, no tendríamos noticia de los aspectos valiosos de la situación, ni seríamos capaces de proyectar idealmente cursos alternativos de acción (Pappas, 20o8: 199). La imaginación relaciona creativamente la experiencia presente con las pasadas, ofreciendo las alternativas posibles que evaluar reflexivamente. De esta manera, la imaginación abre de modo irrestricto las posibilidades de reflexión, posibilitando así el crecimiento moral. De la misma manera que sin imaginación no hay simpatía, sino un mero sentimiento benevolente, sin imaginación no hay evaluación y reflexión moral, sino una mera obediencia mecánica a normas previamente fijadas. 
En última instancia, el crecimiento y la madurez moral no se logran por medio de una razón fría e impasible, sino por medio de la buena voluntad, que implica una mirada comprensiva, imaginativa y simpática. El juicio justo es, tanto para Dewey como para Hannah Arendt, reflexivo, un juicio por el que uno es capaz de tener en cuenta el punto de vista de los otros con el fin de adoptar un concurso de acción (Arendt, 1961: 220-221, en Benhabib, 2006a: 21; Benhabib, 2006b: 232). Pero Dewey dota de contenido moral al juicio político de Arendt, al tiempo que considera que requiere del concurso integrado de la simpatía, la imaginación y la afectividad.

En la medida en que la identificación simpática con los demás fomenta el crecimiento moral, no anula, sino que potencia la propia individualidad. En otras palabras, la imagen que tenemos de nosotros mismos y las posibilidades de nuestra acción están mediatizadas por las relaciones con los otros, por eso la atención a los demás potencia nuestro desarrollo, mientras que el aislamiento lo empobrece.

Si el análisis social de Fraser se centra en las luchas contra la injusticia, que son necesarias, la perspectiva de Dewey lo completa con las actitudes morales imprescindibles para desarrollar en la práctica la paridad participativa. Desarrollar una u otra estrategia, actitudes de lucha o actitudes dialógicas, dependerá del contexto en cuestión. Aunque Dewey no era partidario de luchas violentas sino más bien de reformas educativas con el fin de cambiar actitudes, Dewey aprobaba actuaciones contundentes de las mujeres, si era el camino de que las tomasen en serio (Dewey, 1911: 154).

Dewey hace especial hincapié en la integración de la conducta, que nunca será definitiva; al contrario, los cambios y conflictos la revitalizan. El análisis de Dewey centrado en las transacciones evita reificar la subjetividad o el grupo social; de hecho, las transacciones suponen cambios, pero tampoco mistifica el cambio, ni el conflicto. Los conflictos sociales y personales pueden ser positivos o no, en función de cómo se interpreten y se actúe ante ellos. Si se perpetúan y conducen al choque y aislamiento de posturas, son negativos; pero si se interpretan funcionalmente, es decir, por relación a la conducta, potencian la reflexión y la reorganización dinámica de las transacciones, evitando su mecanización.

En resumen, la perspectiva pragmática de Dewey permite ampliar significativamente el sentido de la justicia de Fraser, atendiendo a los condicionantes de los sujetos que deben aplicar el diálogo normativo. En concreto, Dewey esboza una interpretación funcional de la subjetividad que evita tanto su reificación como su pura deconstrucción. Permite ampliar así el análisis social con 
un análisis de la subjetividad en diálogo con otros marcos teóricos. Esas ampliaciones teóricas refuerzan el potencial crítico de la teoría feminista, pues permiten visibilizar aspectos de la justicia y, por ende, de la igualdad de género, a los que otras teorías son ciegas en pro de una radicalización feminista y democrática.

\section{CONCLUSIONES}

La paridad en la participación que Fraser propone como criterio de justicia y guía de las luchas feministas tiene indudables ventajas. En primer lugar, permite atender a distintas dimensiones de la justicia (económica, cultural y política), sin reduccionismos economicistas o culturalistas. En segundo lugar, y precisamente porque relaciona y distingue distintas dimensiones de las injusticias, su análisis social responde mejor a los distintos ámbitos de dominación de las mujeres en el capitalismo neoliberal, por ejemplo, la explotación, la marginación y el androcentrismo. En tercer lugar, al referir las reivindicaciones feministas a su contexto social, su teoría comprende mejor la relación de las injusticias de género con otro tipo de injusticias de clase, de raza, de etnia, etc.

Su interpretación de la justicia como paridad participativa amplía e integra, desde su punto de vista, los aspectos más interesantes de otros planteamientos: supera el economicismo de los planteamientos marxistas y el culturalismo de las políticas de la identidad integrando sus luchas por la distribución y por el reconocimiento, a las que une las luchas por la representación. De esta manera, su propuesta permite analizar mejor las injusticias y articular medidas en pro de la justicia de género.

Sin embargo, su propuesta tiene límites que frenan su potencial emancipador. Judith Butler, desde presupuestos posestructuralistas, considera que no analiza los condicionamientos institucionales que nos convierten en los sujetos que somos, por ejemplo, la organización social de la sexualidad y el deseo. Honneth señala asimismo que Fraser no tiene en cuenta los condicionantes subjetivos que empujan a los individuos a participar en los movimientos sociales reivindicativos.

El pragmatismo de Dewey permite ampliar el análisis social de Fraser con el análisis de los condicionantes subjetivos de la acción. Ambos coinciden en considerar la democracia como un ideal social que depende de la participación en condiciones de igualdad y pretenden su radicalización, pero Dewey propor- 
ciona un análisis funcional de las transacciones de las que la subjetividad y el orden social son factores. Evita así, por una parte, la reificación de la subjetividad, pero no por ello su dirección reflexiva. Por otra parte, evita la asimilación social, estima los cambios y las diferencias como momentos imprescindibles del desarrollo personal y social, pero sin mistificarlos, como funciones del crecimiento de relaciones participativas.

De esta manera, Dewey amplía la consideración normativa de la paridad en la participación interpretándola no solo como un procedimiento de organización de una sociedad plural, sino como medio de crecimiento personal, como principio que nos permite construir una personalidad autónoma. Atiende así no solo a las reclamaciones sociales, a las luchas, imprescindibles para extender la paridad participativa, sino también a las actitudes personales que requiere y que resultan imprescindibles para la extensión y la radicalización democrática.

\section{BIBLIOGRAFÍA}

Amorós, Celia y De Miguel, Ana (2010). Teoría feminista de la Ilustración a la Globalización. Madrid: Minerva.

Arendt, Hannah (1961). The Crisis in Culture. En Arendt, Hannah. Between Past and Future: Six Exercises in Political Thought. New York: Meridian.

Benhabib, Seyla (2006a). El ser y el otro en la ética contemporánea. Barcelona: Gedisa.

Benhabib, Seyla (2006b). Igualdad y diversidad en la era global. Buenos Aires: Katz.

Benhabib, Seyla, Butler, Judith, Cornell, Drucilla y Fraser, Nancy (2011). Feminist Contentions. New York: Routledge.

Benhabib, Seyla y Cornell, Drucilla (1990). Teoría feminista y teoría crítica. Valencia: Alfons el Magnànim.

Bernstein, Richard (2010). Filosofía y democracia: John Dewey. Barcelona: Herder.

Butler, Judith (2000). Merely Cultural. New Left Review, 2, 109-121.

Butler, Judith (2007). El género en disputa. Barcelona: Paidós. 
Dewey, John (1908). Ethics. En Boydston, Jo Ann (Ed.) (1996). The Collected Works of John Dewey, Middle Works V. Carbondale: Southern Illinois University Press.

Dewey, John (1911). A Symposium on Woman's Suffrage. En Boydston, Jo Ann (Ed.) (1996). The Collected Works of John Dewey, Middle Works VI. Carbondale: Southern Illinois University Press.

Dewey, John (1916). Democracy and Education. En Boydston, Jo Ann (Ed.) (1996). The Collected Works of John Dewey, Middle Works X. Carbondale: Southern Illinois University Press.

Dewey, John (1922). Human Nature and Conduct: An Introduction to Social Psychology. En Boydston, Jo Ann (Ed.) (1996). The Collected Works of John Dewey, Middle Works XIV. Carbondale: Southern Illinois University Press.

Dewey, John (1930). Individualism Old and New. En Boydston, Jo Ann (Ed.) (1996). The Collected Works of John Dewey, Later Works V. Carbondale: Southern Illinois University Press.

Dewey, John (1934). Art as Experience. En Boydston, Jo Ann (Ed.) (1996). The Collected Works of John Dewey, Later Works X. Carbondale: Southern Illinois University Press.

Femenías, María Luisa (2007). El feminismo postcolonial y sus límites.

En Amorós, Celia y de Miguel, Ana (Eds.). Teoría feminista de la Ilustración a la globalización, volumen 3 (155-213). Madrid: Minerva. Foucault, Michel (1990). The Use of Pleasure. The history of sexuality 2. New York: Radom House.

Foucault, Michel (2015). La ética del pensamiento. Para una crítica de lo que somos. Madrid: Biblioteca Nueva.

Fraser, Nancy (1997). Heterosexism, Misrecognition, and Capitalism:

A Response to Judith Butler. Social Text, 52/53, 279-289.

Fraser, Nancy (2000). Rethinking Recognition: Overcoming Displacement and Reification in Cultural Politics. New Left Review, 3, 107-120.

Fraser, Nancy (2011). Dilemas de la justicia en el siglo XXI. Género y globalización. Palma: UIB. 
Fraser, Nancy (2015). Fortunas del feminismo. Quito: IAEN.

Fraser, Nancy y Honneth, Axel (2006). ¿Redistribución o reconocimiento? Madrid: Morata.

Fraser, Nancy y Nicholson, Linda (1988). Social Criticism without Philosophy: An Encounter between Feminism and Postmodernism. Theory, Culture y Society, 5, 373-394.

Hamington, Maurice y Bardwell-Jones, Celia (2012). Contemporary Feminist Pragmatism. New York: Routledge.

Janack, Marianne (2000). Feminist Interpretations of Richard Rorty. Pennsylvania State: University Park Press.

Palacio, Marta (2018). Pragmatismo, emancipación y feminismo. Astrolabio. Revista Internacional de Filosofía, 21, 77-99. doi: 101344/astrolabio2018.21.5.

Pappas, Gregory (2008). John Dewey's Ethics. Democracy as Experience. Indiana: Indiana University Press.

Posada, Luisa (2014). Teoría queer en el contexto español. Reflexiones desde el feminismo. Daimon. Revista Internacional de Filosofía, 63, 147-158. doi: 10.6018/daimon/190041.

Saito, Naoko (1996). Dewey's Idea of Sympathy and Development of Ethical Self: a Japanese Perspective. Philosophy of Education, 1, 389396.

Seigfried, Charlene-Haddock (2001). Feminist Interpretations of John Dewey. University Park: Pennsylvania State University Press. 
